\title{
A Posterior Communicating Segment Aneurysm of the Supraclinoid Internal Carotid Artery Treated with an Extracranial to Intracranial Bypass and Trapping
}

\author{
Burak Ozaydin ${ }^{1}$ \\ Mehmet $\mathrm{C}$ \\ C. Ezgu \\ ${ }^{1}$ Department of Neurological Surgery, University of Wisconsin- \\ Madison School of Medicine and Public Health, Madison, Wisconsin, \\ United States \\ ${ }^{2}$ Department of Neurological Surgery, University of Wisconsin- \\ Madison School of Medicine and Public Health, Madison, Wisconsin, \\ United States
}

J Neurol Surg B Skull Base 2022;83(suppl S3):e616-e618.

\author{
Mustafa K. Baskaya²
}

\begin{abstract}
Keywords

- giant aneurysm

- cerebral bypass

- revascularization

- anterior clinoidectomy
\end{abstract}

Address for correspondence Mustafa K. Baskaya, MD, Department of Neurological Surgery, University of Wisconsin-Madison, 600 Highland Avenue, CSC K8/828, Madison, WI 53792, United States (e-mail: baskaya@neurosurgery.wisc.edu).

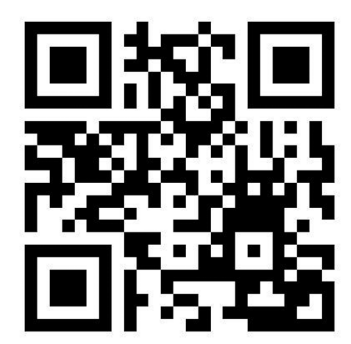

received

April 30, 2020

accepted

October 31, 2020

published online

May 3, 2021
Surgical treatment of giant aneurysms often poses significant challenges. Endovascular techniques have evolved exponentially over the last decades, and most of these complex aneurysms can be treated with flow-diverting techniques; however, successful obliteration of all giant aneurysms is not always possible with endovascular flowdiverting techniques. Although the need for microsurgical intervention has undoubtedly diminished, a versatile-thinking surgeon should keep in mind that obliteration of these aneurysms combined with revascularizing the distal circulation via extracranialintracranial bypass techniques can provide a potentially life-long durable solution. The key to curing these pathologies is to utilize interdisciplinary decision making with a robust knowledge of the pros and cons of different treatment approaches. Herein, we present a case of a giant posterior communicating segment aneurysm of the left supraclinoid internal carotid artery (ICA), which was treated by obliteration (- Fig. 1). Extradural anterior clinoidectomy was used to provide exposure of the supraclinoidal ICA proximal to the aneurysm, and revascularization of the distal circulation was achieved with a common carotid artery to M2-superior trunk bypass using a radial artery interposition graft (-Fig. 2). The patient was a 62-year-old female who presented with vision loss in her left eye but was otherwise neurologically intact. She had a history www.thieme.com/skullbasevideos

www.thieme.com/jnlsbvideos
Dol https://doi.org/ $10.1055 / \mathrm{s}-0041-1725940$ ISSN 2193-6331.

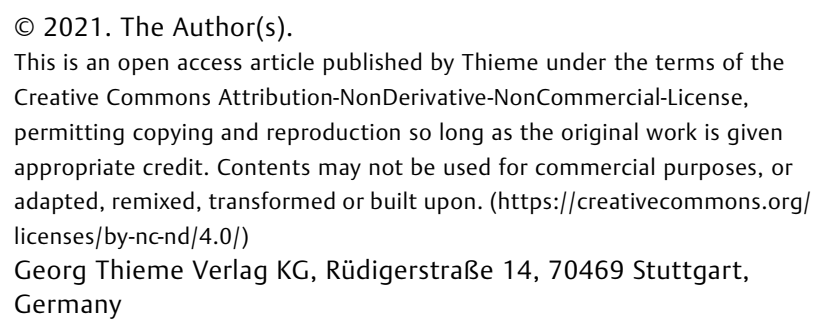


of two unsuccessful flow-diverting stent placement attempts 2 months prior to this surgery. Postoperatively, the patient woke up without any deficits, with her left eye vision partially recovered and ultimately returning to normal at 1-year follow-up. Computed tomography (CT) angiography at a 1-year follow-up showed complete obliteration of the aneurysm and successful revascularization of the distal circulation. The link to the video can be found at: https://youtu.be/Dslul]jj114.

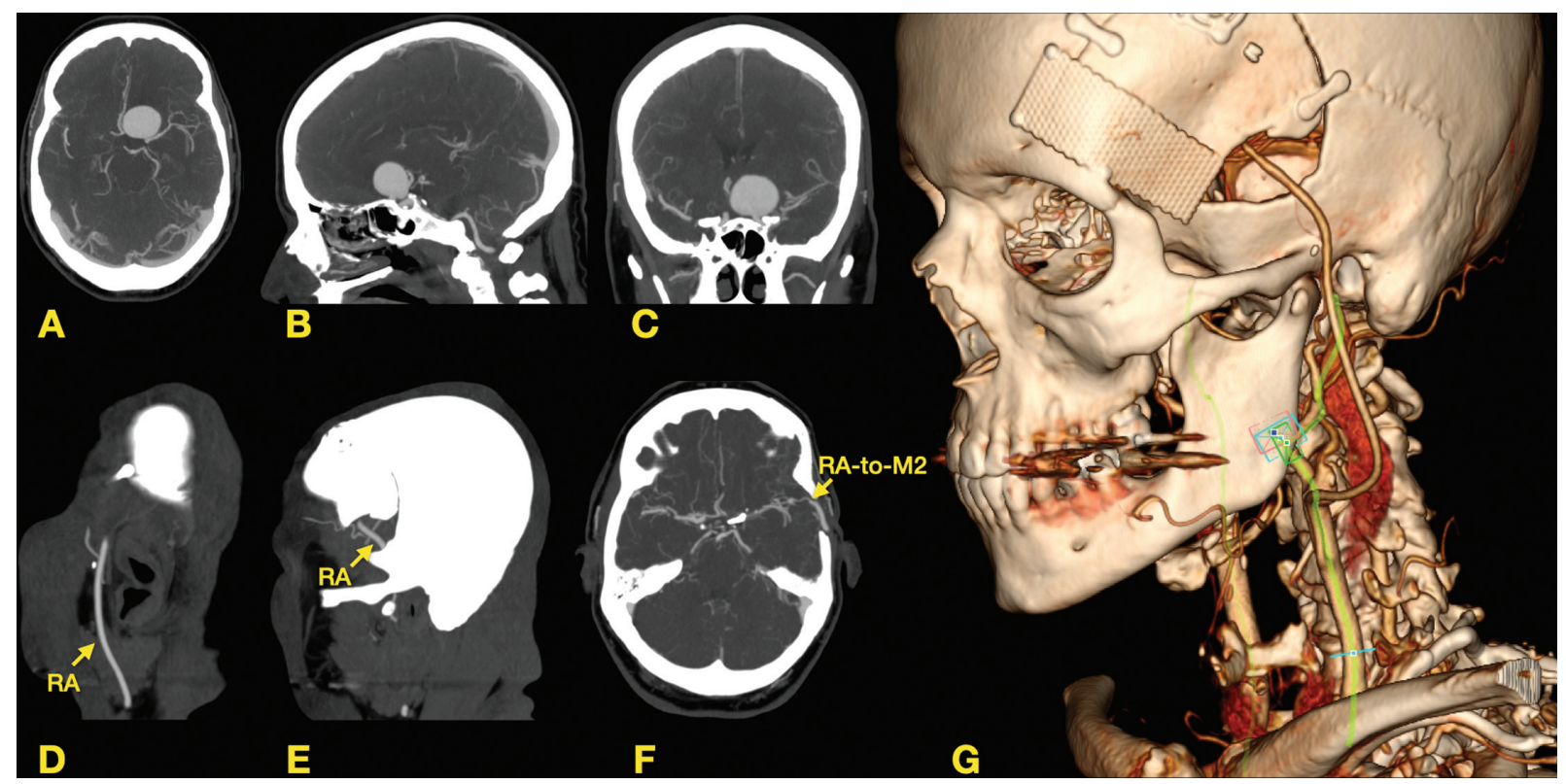

Fig. 1 Preoperative axial (A), sagittal (B), coronal (C), and postoperative sagittal (D, E), axial (F), and 3D reconstructed computerized tomography angiography of the patient. 3D, three-dimensional; RA: radial artery, RA-to-M2: radial artery to M2 superior trunk anastomosis. 


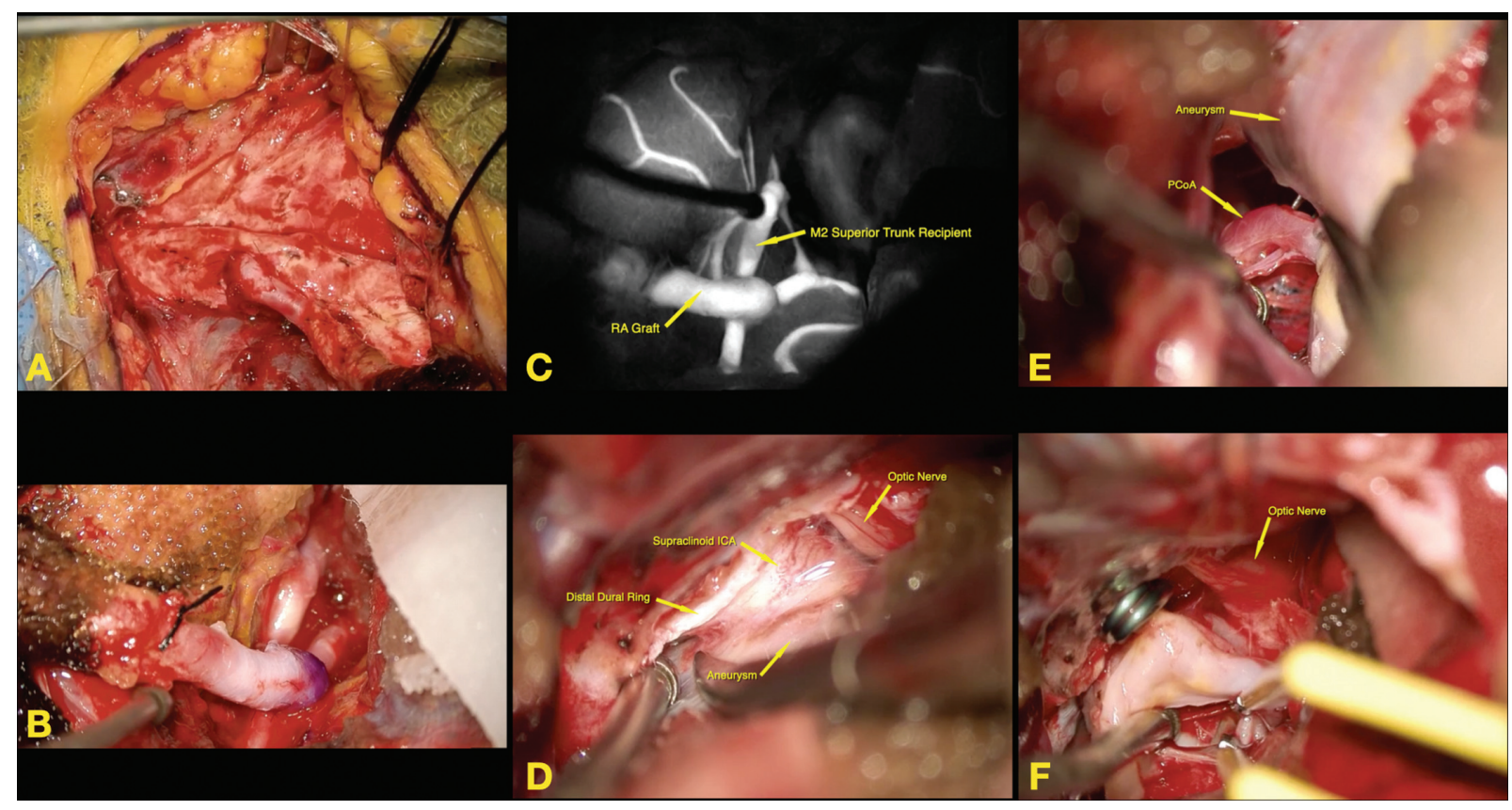

Fig. 2 Intraoperative photomicrographs showing the common carotid artery to radial artery interposition graft anastomosis (A); radial artery to M2 superior trunk anastomosis (B) and photomicrograph of the indocyanine green video angiography (C); view of the supraclinoidal internal carotid artery, distal dural ring, optic nerve, and aneurysm after extradural anterior clinoidectomy (D); the relationship of the aneurysm with the posterior communicating artery $(\mathrm{E})$; decompressed optic nerve after complete trapping and incision of the aneurysm. ICA, internal carotid artery; PCoA, posterior communicating artery.

\section{Note}

This manuscript has not been published before, either as a whole or in part nor is it under consideration for publication elsewhere. Additionally, the authors of this manuscript report no conflicts of interest related to the manuscript, which was not financially supported by any person or institute.

\section{Disclosure}

The authors have no personal, financial, or institutional interest in any of the drugs, materials, or devices described in this article.

\section{Conflict of Interest}

None declared. 